\title{
Computational Techniques Based on Runge-Kutta Method of Various Order and Type for Solving Differential Equations
}

\author{
Vijeyata Chauhan ${ }^{a}$, Pankaj Kumar Srivastava ${ }^{b^{*}}$ \\ Department of Mathematics \\ Jaypee Institute of Information Technology, Noida, India

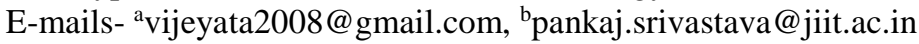 \\ ${ }^{*}$ Corresponding author
}

(Received September 25, 2018; Accepted December 26, 2018)

\begin{abstract}
The Runge-Kutta method is a one step method with multiple stages, the number of stages determine order of method. The method can be applied to work out on differential equation of the type's explicit, implicit, partial and delay differential equation etc. The present paper describes a review on recent computational techniques for solving differential equations using Runge-Kutta algorithm of various order. This survey includes the summary of the articles of last decade till recent years based on third; fourth; fifth and sixth order Runge-Kutta methods. Along with this a combination of these methods and various other type of Runge-Kutta algorithm based articles are included. Comparisons of methods with own critical comments as remarks have been included.
\end{abstract}

Keywords- Runge-Kutta algorithm, Convergence of method, Implicit-Explicit method, Ordinary and partial differential equations.

\section{Introduction}

Several branches of economy, science and engineering frequently contain mathematical models of ordinary differential equations (Arora and Pratiksha, 2019). Only on a few occasions these differential equations have closed form solution otherwise mostly approximate solutions through numerical algorithms arise (Srivastava and Kumar, 2011; Srivastava, 2014). In these numerical tools Runge-Kutta method plays a major role. The present paper covers the application of RungeKutta algorithm to solve ordinary differential equations (ODEs). Special emphasis is given on different forms of Runge-Kutta method.

This article includes here recent papers based on Runge-Kutta method to solve various differential equation systems. The article is structured as follows: Section 2 covers a brief study of Runge-Kutta algorithm. The Section 3 contains the papers having third order Runge-Kutta techniques to solve differential equations. Similarly, in Sections 4, 5 and 6 a description of fourth order, fifth order and sixth order Runge-Kutta techniques to solve differential equations have been summarized. In Section 7, a combination of various order Runge-Kutta techniques is discussed. In Section 8, papers based on several types of Runge-Kutta methods are included. At the end in Section 9, the conclusion and future aspects are discussed.

\section{Runge-Kutta Method}

Runge-Kutta techniques were introduced around 1900 by C. Runge and M. W. Kutta. Then after passage of time this method took a major role in the study of iterative methods based on explicit and implicit which applied to solve ODEs through temporal discretization. 
International Journal of Mathematical, Engineering and Management Sciences

Vol. 4, No. 2, 375-386, 2019

https://dx.doi.org/10.33889/IJMEMS.2019.4.2-030

C. Runge, by completing his famous paper, developed the method of approximating Euler to enhance this scheme which could provide great accuracy, Runge's work was developed by Heun, (1900) and by Kutta (1901).

It is not known for a given order $\mathrm{p}$ what should be the number of stages $\mathrm{s}$ in general. The base of the scheme is expressed as

$\mathrm{y}_{(\mathrm{f}+1)}-\mathrm{y}_{\mathrm{f}}=\sum_{\mathrm{i}=1}^{\mathrm{m}} \mathrm{w}_{\mathrm{i}} k_{i}$

which is difference between the values of $y$ at $\mathrm{t}_{(\mathrm{n})}$ to $\mathrm{t}_{(\mathrm{n}+1)}$ and where $w_{i}{ }^{\prime} s$ are constants and $k_{i}{ }^{\prime} s$ are given by

$\mathrm{k}_{\mathrm{i}}=\mathrm{hf}\left(\mathrm{t}_{\mathrm{n}}+\mathrm{c}_{\mathrm{i}} \mathrm{h}, \mathrm{y}_{\mathrm{n}}+\mathrm{h} \sum_{\mathrm{j}=1}^{\mathrm{i}-1} \mathrm{a}_{\mathrm{ij}} \mathrm{k}_{\mathrm{j}}\right.$

This is the consistency relation of R-K method $\sum_{j=0}^{s} a_{i j}=c_{i}, \sum_{j=0}^{s} b_{i}=1$.

\section{Application of Third Order R-K Method}

(i) The convergence of Euler's method is considered by (Kanagarajan and Sambath, 2010) to improve the ODE problem in a higher convergence of order $\mathrm{O}\left(\mathrm{h}^{3}\right)$ (Ma et al., 1999). It was a development over (Abbasbandy and Viranloo, 2002) algorithms to solve fuzzy based differential (Seikkala, 1987). They have considered

$\left\{u^{\prime}(x)=g(x, u(x)), r \leq x \leq s, y(r)=p\right\}$.

Process produces Backward Differentiation Formula (BDF) format and its fusion shape which joint together to form an obstruct technique. The process is reformulated into a Runge-Kutta type of the same step number $k=3$ to solve (special or general) initial value problem of ODE. They have used collocation of multistep to redefine the derived hybrid method into continuous form to solve $y^{\prime}=f(x, y)$.

(ii) An explicit $3^{\text {rd }}$ order Improved R-K algorithm was described by (Rabieu and Ismail, 2011). They have mentioned that the order of method can be increased through Taylor's series expansion. To increase the efficiency of the method minor amount of function evaluations is required. The broad form in two stages $(s=2)$ is described below

$$
\begin{aligned}
& u_{n+1}=u_{n}+h\left(a_{1} L_{1}-a_{-1} L_{-1}+a_{2}\left(L_{2}-L_{-2}\right),\right. \\
& L_{1}-g\left(x_{n}, u_{n}\right)=0, L_{-1}-g\left(x_{n-1}, u_{n-1}\right)=0, \\
& L_{2}-g\left(x_{n}+c_{2} h, u_{n}+a_{21} h, L_{1}\right)=0, L_{-2}-g\left(x_{n-1}+c_{2} h,, u_{n-1}+a_{21} h, L_{-1}\right)=0
\end{aligned}
$$

where $0 \leq c \leq 1 \quad c_{i}=\sum_{j=1}^{i-1} a_{i j}$ which is called row sum condition here $c_{2}=a_{21}$. 
International Journal of Mathematical, Engineering and Management Sciences

Vol. 4, No. 2, 375-386, 2019

https://dx.doi.org/10.33889/IJMEMS.2019.4.2-030

(iii) In 2009, Ababneh with Ahmad and Ismail introduced a new scheme of solving IVPs by R-K methods (Ababneh et al., 2009). In this method using harmonic mean and approximations of $f^{\prime}$ they replaced evaluation of $f$.The author considered $y_{n+1}=y_{n}+\left[2 \frac{k_{1} k_{2}}{k_{1}+k_{2}}\right]$, where stages are given by

$k_{1}=h f\left(x_{n}\right), k_{2}=h\left(f\left(y_{n}+a_{21} k+a_{22} h\left(f\left(y_{n}-f\left(y_{n-1}\right)\right)\right)\right.\right.$.

The Taylor series expansion of $k_{1}$ and $k_{2}$ have been used in (1) to obtain an expression of $y_{n+1}$ (Ademiluyi and Babatola, 2001). The authors also proposed the stability analysis.

(iv) In a study of hybrid fuzzy system, Runge-Kutta Nystrom method of order three over hybrid fuzzy differential equations was introduced (Saveetha and Pandian, 2012). Convergence of result is stated and discussed the complete error analysis using numerical examples. The fuzzy hybrid equation under condition is

$u^{\prime}(x)=g\left(x, u(x), \lambda_{k}, u\left(x_{k}\right)\right), x \in\left[x_{k}, x_{k+1}\right], u_{k}=u\left(x_{k}\right)$

where' stands for Seikkala derivative.

The R-K Nystrom algorithm of third order is developed to solve (4) and it is assumed that the solution of (4) satisfies the properties of existence and the uniqueness for every interval $\left[t_{k}, t_{k+1}\right]$. Further the authors have replaced the system by a system equivalent to (4) as

$$
\left\{\begin{array}{l}
u^{\prime}(x)-g\left(x, u, \lambda_{k}\left(u_{k}\right)\right)=0 \equiv F_{k}(x, \underline{u}, \bar{u}), u\left(x_{k}\right)-\underline{u_{k}=0} \\
\overline{u^{\prime}}(x)-\bar{g}\left(x, u, \lambda_{k}\left(u_{k}\right)\right)=0 \equiv G_{k}(x, \underline{u}, \bar{u}), \bar{u}\left(x_{k}\right)-\overline{u_{k}}=0
\end{array}\right\}
$$

A Comparison with the Euler (Pederson and Sambandham, 2007) and Runge Kutta Nystrom method (Kanagarajan and Sampath, 2010) is discussed in the paper.

(v) In a recent study Runge-Kutta (R-K) 3 stage geometric mean based algorithm is applied on autonomous IVP by (Chauhan and Srivastava, 2018). It contains methodology with convergence analysis. The accuracy and efficiency is confirmed through numerical illustration. The outputs are compared to other techniques and proposed methodology is found efficient.

\section{Application of Fourth Order R-K Method}

(i) Fuzzy differential system has become an interesting field these days and the fourth order R-K method has been found as a useful technique to unravel such fuzzy differential equation (Parandin, 2014). It is applied over $2^{\text {nd }}$ order differential equation

$\frac{d^{2} x}{d t^{2}}=f\left(t, x, \frac{d x}{d t}\right), x\left(t_{0}\right)=x_{0}, x^{\prime}\left(t_{0}\right)=y\left(t_{0}\right)=y_{0}$

which is reduced to second order simultaneous fuzzy differential equation. 
International Journal of Mathematical, Engineering and Management Sciences

Vol. 4, No. 2, 375-386, 2019

https://dx.doi.org/10.33889/IJMEMS.2019.4.2-030

$$
\left\{\begin{array}{l}
\frac{d x}{d t}=y=f_{1}(t, x, y), t \in\left(t_{0}, T\right) \\
\frac{d x}{d t}=f_{2}(t, x, y)
\end{array}\right\}, x\left(t_{0}\right)=x_{0}, x^{\prime}\left(t_{0}\right)=y_{0}
$$

where $x_{0}, y_{0}$ are fuzzy number. Approximate solution of the above equations is found through suitable application of $\mathrm{R}-\mathrm{K}$ technique.

(ii) An algorithm to solve fuzzy initial value problem based on the fourth order R-K method is proposed in detail by (Sharmila and Amritharaj, 2013). It was established that in the new method, order of convergence is $\mathrm{O}\left(\mathrm{h}^{4}\right)$. This was observed that the proposed method suited very well to solve linear and non- linear initial value fuzzy problems. The authors considered the IVP

$\frac{d y}{d t}=f(t, y(t)) ; a \leq t \leq b$

$y(a)=\alpha$, and solution is specified by

$y_{n+1}=y_{n}+h \sum_{i=1}^{3}$ means

where means includes Contra harmonic Mean (CoM), which involves $k_{i}$, for $1 \leq i \leq 4$, where

$k_{1}=f\left(t_{n}, y_{n}\right), k_{2}=f\left(t_{n}+a_{1} h, a_{1} h k_{1}\right), k_{3}=f\left(t_{n}+\left(a_{2}+a_{3}\right) h, y_{n}+a_{2} h k_{1}+a_{3} h k_{2}\right.$,

$k_{4}=f\left(t_{n}+\left(a_{4}+a_{5}+a_{6}\right) h, y_{n}+a_{4} h k_{1}+a_{5} h k_{2}+a_{6} h k_{3}\right.$.

The fourth order formulae based on Runge-Kutta scheme using Contra harmonic Mean is as follows

$$
\begin{aligned}
& y_{n+1}=y_{n}+\frac{h}{3}\left(\frac{k_{1}^{2}+k_{2}^{2}}{k_{1}+k_{2}}+\frac{k_{2}^{2}+k_{3}^{2}}{k_{2}+k_{3}}+\frac{k_{3}^{2}+k_{4}^{2}}{k_{3}+k_{4}}\right) \\
& a=t_{0} \leq t_{1} \leq \ldots \leq t_{N}=b, h=\frac{b-a}{N}=t_{i+1}-t_{i} .
\end{aligned}
$$

(iii) In 2015, R-K scheme of order 4 was applied to solve some standard initial value problems (Islam, 2015). The efficiency and accuracy of the method was checked using numerical examples. Error analysis and stability of the method was discussed. The standard form of order 4 method is $y_{n+1}=y_{n}+\frac{1}{6}\left(k_{1}+k_{2}+k_{3}+k_{4}\right)$,

where $k_{1}=h f(x, y), k_{2}=h f\left(x+\frac{h}{2}, y+\frac{k_{1}}{2}\right), k_{3}=h f\left(x+\frac{h}{2}, y+\frac{k_{2}}{2}\right), k_{4}=h f\left(x+h, y+k_{4}\right)$. 
International Journal of Mathematical, Engineering and Management Sciences

Vol. 4, No. 2, 375-386, 2019

https://dx.doi.org/10.33889/IJMEMS.2019.4.2-030

The convergence of the IVP is intended by $e_{n}=\mid y\left(x_{n}-y_{n} \mid<\delta\right.$ where $y\left(x_{n}\right)$ is approximate solution, $y_{n}$ is exact solution and $\delta$ depends on the problem. Absolute error is given by $e_{n}$.

\section{Remarks}

To achieve the desired accuracy the step size has to be very small.

The numerical results obtained agreed with exact solution to good extent.

2D incompressible flow (Kaushik, 2019; Ahuja and Gupta, 2019; Gunwant, 2019; Ramadevi, Ramana and Sugunamma, 2019) also have a similar impact in computational fluids.

\section{Application of Fifth Order R-K Method}

(i) Jayakumar et al. (2012) have found the approximate solution of fuzzy differential equations of Nth-order. In such approach the equation was based on Seikkala's derivative with IVP. The authors have applied fifth order Runge-Kutta algorithm to approximate the problem under consideration. The convergence was described along with the stability of the method. This algorithm is applicable to obtain fuzzy solution, which was well justified through certain examples.

\section{Application of Sixth Order R-K Method}

(i) An efficient sixth order three implicit R-K method has been established by Agam and Yahaya to solve initial value problem of first order (Agam and Yahaya, 2014). To derive continuous schemes Collocation method was used which has given higher order schemes. In this algorithm types of implicit approaches under discussion were

Singly implicit approach with order $p=s$ (s = number of stages)

Diagonally implicit methods

Multiply implicit methods with order $p=2 s$.

\section{Remarks}

$>$ Implicit R-K approach based on Gaussian quadrature have order $=2 s, \mathrm{~s}=$ number of stages,

The propose scheme satisfies Runge-Kutta conditions $\sum_{j=0}^{3} a_{i j}=c_{i}, \sum_{i=1}^{3} b_{i}=1$.

\section{Application of Multiple Order R-K Method}

(i) In 2009 Butcher has described how R-K schemes up to $4^{\text {th }}$ order assure precisely the same parameters as for a general system of high-dimension (Butcher, 2009). Using the equation

$$
y_{i}=y_{0}+h \sum_{j=1}^{s} a_{i j} f_{j}, i=1,2, \ldots, s
$$

for a random technique, each phase rate was calculated from the original rate and the $\mathrm{s}$ phase derivatives. In this approach only explicit method was considered with summation $j \leq i-1$. 
International Journal of Mathematical, Engineering and Management Sciences

Vol. 4, No. 2, 375-386, 2019

https://dx.doi.org/10.33889/IJMEMS.2019.4.2-030

\section{Remarks}

$>$ Order scheme was introduced by Kutta, there was errors in the arrangement of results, partially approved by Nystrom (Nystrom and Uber, 1925) and derived explicit fifth order methods which is easy (Butcher, 2009).

$>$ The primary explicit technique of $6^{\text {th }}$ order was established by Huta (Huta,1956). It was assumed that eight stages would be necessary.

(ii) An additive Runge-Kutta algorithm of orders varying from two to four has been developed by (Liu and Zou, 2006). It was applied to solve some nonlinear system of Ordinary differential Equations.

The proposed algorithm is especially applicable for nonlinear PDEs with constraint. Linear ODEs (Srivastava et al., 2012) or PDEs may be solved with the proposed method at each time step.

(iii) R-K method of fourth and fifth order was developed for the solution of dependency problem in fuzzy computation (Kanagarajan et al., 2014. The equation under consideration was

$$
\begin{aligned}
& \frac{d x}{d t}=f(t, x(t)) ; \quad \text { but with crisp initial condition } x\left(t_{0}\right)=x_{0} \in R \text { and } \quad t \in\left[t_{0}, T\right] \\
& x_{r+1}=x_{r}+h \sum_{j=1}^{m} b_{j} k_{j}^{(1)}+h^{2} \sum_{j=1}^{m} c_{j} k_{j}^{(2)} \\
& k_{j}^{(1)}=f\left(t_{r}, y_{r}+h \sum_{j=1}^{s-} a_{j s} k_{j}^{(1)}\right. \\
& k_{j}^{(2)}=f^{\prime}\left(t_{r}, y_{r}+h \sum_{s=1}^{j-} b_{j s} k_{s}^{(1)}\right.
\end{aligned}
$$

\section{Application of Various Other type of R-K Method}

(i) Gottlieb has presented algorithm to solve hyperbolic PDEs through lines approximations using semi-discrete stability of time discretization (Gottlieb, 2005). The author described the connection of the time step limit on SSP schemes with the assumption of contractivity and monotonicity development of SSP techniques and the recently developed theory. He has also considered the optimal explicit SSP algorithms to solve linear problems and nonlinear problems.

(ii) In 2008, two steps accelerated Runge-Kutta method has found its place in literature (Udwadia and Farahani, 2008). This method has found to achieve the same order of local accuracy efficiently, as for orders three, four, and five; they require only two, three, and five function assessments per time stride, respectively. This is observed that R-K method of 3, 4 and 5 order require three, four, and six function evaluations per time step of integration, respectively (Runge, 1895; Kutta, 1901).

Some of the important significance of the paper is

The cases covered are for $\mathrm{v}=2, \ldots, 5$. As for $\mathrm{v}=1,2^{\text {nd }}$ order ARK2 scheme is possible.

For this purpose they used three techniques

a) minimization of a common bound on the inaccuracy 
International Journal of Mathematical, Engineering and Management Sciences

Vol. 4, No. 2, 375-386, 2019

https://dx.doi.org/10.33889/IJMEMS.2019.4.2-030

b) minimization of a norm of the error (Dormand and Prince, 1980)

c) satisfaction as many entity order equations as probable (Byrne and Lambert, 1966).

(iii) Akanbi has increased the efficiency of explicit R-K methods by increasing terms in incremental function expanded by the Taylors' series (Akanbi, 2011). The equation under consideration

$y^{\prime}(x)=f(x, y(x)), x \in\left[x_{0}, x_{\text {end }}\right], y\left(x_{0}\right)=y_{0}$

has been solved by the GERK (Geometric explicit R-K method).

General form is

$$
y_{n+1}-y_{n}=\Phi_{G}\left(x_{n}, y_{n} ; h\right)
$$

where

$$
\begin{aligned}
& \Phi_{G}\left(x_{n}, y_{n} ; h\right)=b_{1} \sqrt{k_{1} k_{2}}+b_{2} \sqrt{k_{2} k_{3}} \\
& k_{1}=f\left(y_{n}\right), k_{2}=f\left(y_{n}+a_{21} k_{1}\right), k_{3}=f\left(y_{n}+a_{31} k_{1}+a_{32} k_{2} .\right.
\end{aligned}
$$

The GERK technique was based on increment function $\Phi_{G}\left(h_{n} ; h\right)=h f+\frac{1}{2} h^{2} f f_{y}+h^{3}\left(f f_{y}^{2}+f^{2} f_{y y}\right)$.

The assets of the function $\Phi_{G}\left(h_{n} ; h\right)$ are very important and can be established in proving stability and convergence characteristics.

\section{Remarks}

This scheme is linear in $y_{n}$ along with $f_{n}$, and is compared with the method of Nystrom, Heun, Kutta, optimal/Raltson, 3sGRK_1, 3sGRK_2 (Lambert, 1991; Lee, 2004).

(iv) Ghanaie has interpreted a FDE by means of the robustly generalized concept of differentiability (Ghanaie, 2011). Using this concept the author has tried to get two approximations of FDE and expression of the generalized R-K approximation scheme of order 2 and analyzed its inaccuracy. The author solved an illustration in the nuclear decay form equation to demonstrate that how this method is competent with other methods.

(v) First and second order derivation based 3stage R-K method has been established by (Wusu et al., 2013). The authors have analyzed stability along with consistency of the algorithm and compared the accuracy of the proposed method using some examples. They considered the first order IVP

$u^{\prime}(t)=g(t, u), \quad u\left(t_{0}\right)=u_{0}$ and general form of it is specified by $u_{n+1}=u_{n}+h \Phi_{G}\left(t_{n}, u_{n} ; h\right)$ 
International Journal of Mathematical, Engineering and Management Sciences

Vol. 4, No. 2, 375-386, 2019

https://dx.doi.org/10.33889/IJMEMS.2019.4.2-030

The properties of the incremental function $\Phi_{G}\left(t_{n}, u_{n} ; h\right)$ are very critical to its convergence and stability (Fatunla, 1988; Butcher, 2000; Akanbi and Okunuga, 2005; Akanbi, 2011).

(vi) The study of time discretization of differential equations in nonlinear parabolic form has been done through R-K algorithm (Ostermann and Thalhammer, 2002). For analysis the property of linearization as uniform operator has been used. The authors have derived smooth and nonsmooth error and proved convergence for stable R-K methods. They have used some numerical examples to estimate the convergence. The concept is based on $v(t)$ to get $v^{\prime}(t)=C(t) v(t)+g(t, v(t)), v(0)=v_{0}$. Authors have claimed that the R-K methods are invariant under the proposed linearization.

(vii) Considering a d-dimensional equation of the form

$\frac{d x(t)}{d t}=f(t, x(t)), t \in\left[t, T_{0}\right], x\left(t_{0}\right)=x_{0}$

having $x_{0} \in D$ and $f:\left[t_{0}, T\right] \times D \rightarrow R_{d}$ a differentiable function R-K method have been applied (Cruz et al., 2013). In this for $h>0$, a partition of $T$ was established as $t_{0}<t_{1}<\ldots<t_{N}=T$ of the time interval $\left[t, T_{0}\right]$.

It was notable that the local linear ODE has solution which is obtained through the solution of the local nonlinear ODE

$$
\frac{d x(t)}{d t}=f(t, z(t)), t \in\left[t_{n}, t_{n+1}\right], \quad z\left(t_{n}\right)=y_{n}
$$

(viii) The concept of GDN-stability and D-convergence along with R-K approach has been established by (Yuan et al., 2012). The authors have discussed that D-convergence is obtained by algebraic stability. To prove theoretical result a numerical example was given. Here the authors have considered

$$
y^{\prime}(t)=\sum_{m=1}^{m} f^{m}\left(t, y(t), y\left(t-t_{m}\right), \int_{t-m}^{t} g^{(m)}(t, s, y(s)) d s\right),
$$

$t \in\left[t_{0}, T\right], y(t)=\phi(t), t \in\left[t_{0}-\tau, t_{0}\right]$, where

$\tau_{1} \leq \tau_{2} \leq \ldots \leq \tau_{m}=\tau, f^{(v)}:\left[t_{0}, T\right] \times C^{N} \times C^{N} \rightarrow C^{N}, v=1,2, \ldots, m, \phi:\left[t_{0}-\tau, t_{0}\right] \rightarrow C^{N} \quad$ are continuous functions such that the problem under consideration has unique solution. 
International Journal of Mathematical, Engineering and Management Sciences

Vol. 4, No. 2, 375-386, 2019

https://dx.doi.org/10.33889/IJMEMS.2019.4.2-030

(ix) Consider the nonlinear FDFE

$u^{\prime}(x)-f(x, u(x), u(x-\tau))=0$,

$z(t)=g(x, u(x) \cdot u(x-\tau), z(x-\tau), u(x)-\phi(x)=z(x)-\psi(x)=0, x \geq 0$

where $\tau>0$ is a real constant, yand zare unknown vectors of complex functions, $f$ and $g$ are given vectors of complex functions, and $\phi$, satisfying $(0)=(0,(0), \phi(-\tau), \psi(-\tau))$. In 2014 the approximation of (21) has been developed through R-K scheme (Yu et al., 2014).

(x) Yuan and Song have discussed the convergence and stability of two-step Runge-Kutta methods for solving nonlinear Volterra delay integro-differential equations (Yuan and Song, 2013). The concept of D-convergence was applied for the convergence. In this document authors have considered

$$
y^{\prime}(t)=p\left(t, y(t), y(t-k), \int_{t-k}^{t} q(t, v, y(v) d v, t \in[0, T], \quad y(t)=\omega(t) t \in[-k, 0]\right.
$$

with $p, q$ and $\omega$ are smooth enough, such that it has an exclusive result $y(t)$ and $k$ is a positive delay term.

\section{Conclusion}

The Runge-Kutta methods fall under one step method with multiple stages. However stage numbers and order of the method has no generalized common relation above order four. The method is applicable to solve differential equation of the type's explicit, implicit, partial differential equation and delay differential equation etc. The method is helpful in studying the solution of differential equations which have wide applications in daily life. The convergence, stability analysis of methods are discussed in the above reviewed papers and found that the R-K method is competing well with other methods for the solution of these types of equations. The method is efficient with good accuracy.

\section{Conflict of Interest}

Both authors have contributed equally in this work. The authors declare that there is no conflict of interest for this publication.

\section{Acknowledgments}

The authors extend their appreciation to the anonymous reviewers for their valuable suggestions.

\section{References}

Ababneh, O. Y., Ahmad, R., \& Ismail, E. S. (2009). New multi-step Runge-Kutta method. Applied Mathematical Sciences, 3(45), 2255-2262.

Abbasbandy, S., \& Viranloo, T. A. (2002). Numerical solutions of fuzzy differential equations by Taylor method. Computational Methods in Applied Mathematics, 2(2), 113-124. 
International Journal of Mathematical, Engineering and Management Sciences

Vol. 4, No. 2, 375-386, 2019

https://dx.doi.org/10.33889/IJMEMS.2019.4.2-030

Ademiluyi, R. A., \& Babatola, P. O. (2001). Semi implicit rational Runge-Kutta formulas of approximation of stiff initial value problems in ODEs. Journal of Mathematical Science and Education, 3, 1-25.

Agam, S. A., \& Yahaya, Y. A. (2014). A highly efficient implicit Runge-Kutta method for first order ordinary differential equations. African Journal of Mathematics and Computer Science Research, 7(5), $55-60$.

Ahuja, J., \& Gupta, U. (2019). Rayleigh-Bénard convection for nanofluids for more realistic boundary conditions (rigid-free and rigid-rigid) using darcy model. International Journal of Mathematical, Engineering and Management Sciences, 4(1), 139-156.

Akanbi, M. A. (2011). On 3-stage geometric explicit Runge-Kutta method for singular autonomous initial value problems in ordinary differential equations. Computing, 92(3), 243-263.

Akanbi, M. A., \& Okunuga, S. A. (2005). On region of absolute stability and convergence of 3-stage multiderivative explicit Runge-Kutta methods. Journal of the Sciencea Research and Development Institute, 10, 2005-2006.

Arora, G., \& Pratiksha (2019). A cumulative study on differential transform method. International Journal of Mathematical, Engineering and Management Sciences, 4(1), 170-181.

Butcher, J. C. (2000). Numerical methods for ordinary differential equations in the 20th century. Journal of Computational and Applied Mathematics, 125(1-2), 1-29.

Butcher, J. C. (2009). On fifth and sixth order explicit Runge-Kutta methods: order conditions and order barriers. Canadian Applied Mathematics Quarterly, 17(3), 433-445.

Butcher, J. C. (2016). Numerical methods for ordinary differential equations. John Wiley \& Sons.

Byrne, G. D., \& Lambert, R. J. (1966). Pseudo-Runge-Kutta methods involving two points. Journal of the ACM, 13(1), 114-123.

Chauhan, V., \& Srivastava, P. K. (2018). Trio-Geometric mean-based three-stage Runge-Kutta algorithm to solve initial value problem arising in autonomous systems. International Journal of Modeling, Simulation, and Scientific Computing, 9(04), 1850026.

de la Cruz, H., Biscay, R. J., Jiménez, J. C., \& Carbonell, F. (2013). Local linearization-Runge-Kutta methods: A class of A-stable explicit integrators for dynamical systems. Mathematical and Computer Modelling, 57(3-4), 720-740.

Dormand, J. R., \& Prince, P. J. (1980). A family of embedded Runge-Kutta formulae. Journal of Computational and Applied Mathematics, 6(1), 19-26.

Fatunla, S. O. (1988). Numerical methods for initial value problems in ordinary differential equations. Academic Press. ISBN: 9781483269269.

Ghanaie, Z. A., \& Moghadam, M. M. (2011). Solving fuzzy differential equations by Runge-Kutta method. The Journal of Mathematics and Computer Science, 2(2), 208-221.

Gottlieb, S. (2005). On high order strong stability preserving Runge-Kutta and multi step time discretizations. Journal of Scientific Computing, 25(1-2), 105-128.

Gunwant, D. (2019). Stress concentration studies in flat plates with rectangular cut-outs using finite element method. International Journal of Mathematical, Engineering and Management Sciences, 4(1), 66-76.

Heun, K. (1900), Neue methoden zur approximativen integration der differentialgleichungen eincr unable ver'anderliehen. Zeitschrift für angewandte Mathematik und Physik, 45, 23-38.

Huta, A. (1956). Une amélioration de la méthode de Runge-Kutta-Nystrôm pour la résolution numérique des équations différentielles du premier ordre. Acta Mathematica Universitatis Comenianae, 1, 201-224. 
International Journal of Mathematical, Engineering and Management Sciences

Vol. 4, No. 2, 375-386, 2019

https://dx.doi.org/10.33889/IJMEMS.2019.4.2-030

Islam, M. A. (2015). Accurate solutions of initial value problems for ordinary differential equations with the fourth order Runge Kutta method. Journal of Mathematics Research; Canadian Center of Science and Education, 7(3), 41-45.

Jayakumar, T., Kanagarajan, K., \& Indrakumar, S. (2012). Numerical solution of nth-order fuzzy differential equation by Runge-Kutta method of order five. International Journal of Mathematical Analysis, 6(58), 2885-2896.

Kanagarajan, K., \& Sambath, M. (2010). Runge-Kutta Nystrom method of order three for solving fuzzy differential equations. Computational Methods in Applied Mathematics, 10(2), 195-203.

Kanagarajan, K., Muthukumar, S., \& Indrakumar, S. (2014). Numerical solution of fuzzy differential equations by extended Runge-Kutta method and the dependency problem. International Journal of Mathematics Trends and Technology, 6, 113-122.

Kaushik, A. (2019). Numerical study of 2nd incompressible flow in a rectangular domain using chorin's projection method at high Reynolds number. International Journal of Mathematical, Engineering and Management Sciences, 4(1), 157-169.

Kutta, W. (1901). Beitrag zur näherungsweisen integration totaler differentialgleichungen. Zeitschrift für Mathematik und Physik, 46, 435-453.

Lambert, J. D. (1991). Numerical methods for ordinary differential systems: the initial value problem. John Wiley \& Sons, ISBN: 978-0-471-92990-1.

Lee, J. H. J. (2004). Numerical methods for ordinary differential equations: a survey of some standard methods (Doctoral dissertation, ResearchSpace@ Auckland).

Liu, H., \& Zou, J. (2006). Some new additive Runge-Kutta methods and their applications. Journal of Computational and Applied Mathematics, 190(1-2), 74-98.

Ma, M., Friedman, M., \& Kandel, A. (1999). Numerical solutions of fuzzy differential equations. Fuzzy Sets and Systems, 105(1), 133-138.

Nyström, E. J. (1925). Über die numerische Integration von Differentialgleichungen: (Mitgeteilt am 23. Sept. 1925 von E. Lindelöf und KF Sundman). SocietasscientiarumFennica.

Ostermann, A., \& Thalhammer, M. (2002). Convergence of Runge-Kutta methods for nonlinear parabolic equations. Applied Numerical Mathematics, 42(1-3), 367-380.

Parandin, N. (2014). Numerical solution of fuzzy differential equations of 2nd-order by Runge-Kutta method. Journal of Mathematical Extension, 7(3), 47-62.

Pederson, S., \& Sambandham, M. (2007). Numerical solution to hybrid fuzzy systems. Mathematical and Computer Modelling, 45(9-10), 1133-1144.

Rabieu, F., \& Ismail, F. (2011). Third order improved Runge-Kutta method for solving ordinary differential equation. International Journal of Applied Physics and Mathematics, 1(3), 191-194.

Ramadevi, B., Ramana, R. J. V., \& Sugunamma, V. (2019). Influence of thermo diffusion on time dependent casson fluid flow past a wavy surface. International Journal of Mathematical, Engineering and Management Sciences, 3(4), 472-490.

Runge, C. (1895). Über die numerische auflösung von differentialgleichungen. Mathematische Annalen, 46(2), 167-178.

Saveetha, N., \& Pandian, S. C. (2012). Numerical solution of fuzzy hybrid differential equation by third order Runge Kutta Nystrom method. Mathematical Theory and Modeling, 2(4), 8-17.

Seikkala, S. (1987). On the fuzzy initial value problem. Fuzzy Sets and Systems, 24(3), 319-330. 
International Journal of Mathematical, Engineering and Management Sciences

Vol. 4, No. 2, 375-386, 2019

https://dx.doi.org/10.33889/IJMEMS.2019.4.2-030

Sharmila, R. G., \& Amritharaj E.C. H. (2013). Numerical solution of nth order fuzzy initial value problems by fourth order Runge-Kutta method based on centroidal mean. IOSR Journal of Mathematics, 6(3), 47-63.

Srivastava, P. K. (2014). Study of differential equations with their polynomial and nonpolynomial spline based approximation. Acta Technica Corviniensis-Bulletin of Engineering, 7(3), 139.

Srivastava, P. K., \& Kumar, M. (2011). Numerical treatment of nonlinear third order boundary value problem. Applied Mathematics, 2(08), 959.

Srivastava, P. K., Kumar, M., \& Mohapatra, R. N. (2012). Solution of fourth order boundary value problems by numerical algorithms based on nonpolynomial quintic splines. Journal of Numerical Mathematics and Stochastics, IV, 13-25.

Udwadia, F. E., \& Farahani, A.(2008). Accelerated Runge-Kutta methods. Discrete Dynamics in Nature and Society, Article ID 790619, 38 pages. http://dx.doi.org/10.1155/2008/790619.

Wusu, A. S., Akanbi, M. A., \& Okunuga, S. A. (2013). A three-stage multiderivative explicit Runge-Kutta method. American Journal of Computational Mathematics, 3(02), 121-126.

Yu, Y., Liu, Z., \& Wen, L. (2014). Stability analysis of Runge-Kutta methods for nonlinear functional differential and functional equations. Journal of Applied Mathematics, Article ID 607827, 9 pages http://dx.doi.org/10.1155/2014/607827.

Yuan, H., \& Song, C. (2013). Nonlinear stability and convergence of two-step Runge-Kutta methods for Volterra delay integro-differential equations. Abstract and Applied Analysis, Article ID 683137, 14 pages, http://dx.doi.org/10.1155/2013/683137.

Yuan, H., Zhao J., \& Xu, Y. (2012). Nonlinear stability and D-convergence of additive Runge-Kutta methods for multidelay-integro-differential equations. Abstract and Applied Analysis, Article ID 854517, 22 pages, http://dx.doi.org/org/10.1155/2012/854517. 